Pacific Journal of Mathematics

APPROXIMATION AND HARMONIC CONTINUATION OF
AXIALLY SYMMETRIC POTENTIALS IN $E^{3}$ 


\title{
APPROXIMATION AND HARMONIC CONTINUATION OF AXIALLY SYMMETRIC POTENTIALS IN $E^{3}$
}

\author{
Peter A. McCoy
}

Let $F=F(x, y)$ be a complex valued axisymmetric potential (ASP) regular in the closed unit sphere about the origin in $E^{3}$. Let the error in the approximation of $F$ over $\mathscr{R}_{n, \nu}$ (where $\mathscr{R}_{n, \nu}$ is the set of all Newtonian potentials $R_{n, \nu} \equiv P_{n}^{*}\left(1 / Q_{\nu}\right)$ generated from axisymmetric harmonic polynomials $P_{n}$ and $Q_{\nu}$ by quasimultiplication) be defined by

$E_{n, \nu}(F)=\inf \left\{\sup \left\{\left|F(x, y)-R_{n, \nu}(x, y)\right|: x^{2}+y^{2}=1\right\}: R_{n, \nu} \in \mathscr{R}_{n, \nu}\right\}$ $n, \nu=0,1,2, \cdots$. Then properties of the sequence $\left\{\rho_{\nu}\right\}_{\nu=0}^{\infty}$, $\rho_{\nu}^{-1}=\lim \sup _{n \rightarrow \infty}\left[E_{n, \nu}(F)\right]^{1 / n}$, determine:

(i) the sphere to which $F$ continues as an ASP with atmost (precisely) $\nu$-singular circles (ii) the largest sphere of continuation as an ASP and (iii) the NASC for a sphere to contain infinitely many singular circles of the continuation of $F$.

These results employ the Bergman and Gilbert Integral Operator Method to extend classical theorems from function theory due to J. L. Walsh and E. B. Saff.

1. Introduction. The classical theorem from function theory of J. Hadamard $[5,8]$ determines the number of (free) singularities of an analytic function in a disk from its' Taylor's series coefficients. This determination extends to generalized axisymmetric potentials (GASP) expanded as Fourier series of Zonal Harmonics on $E^{2 \alpha}\left(E^{2 \alpha}=E^{k}\right.$, $2 \alpha=k-3, k=3,4, \cdots)$ by a theorem of R. P. Gilbert [6, 7] who used integral operator methods to "transplant" the Hadamard theorem, thereby determining the number of (free) singular circles of the GASP in a hypersphere of fixed radius from its' Fourier series coefficients.

The theorems from function theory of E. B. Saff [16] and J. L. Walsh $[16,18,19]$ focus on the relation of the rational approximation of an analytic function, regular on a closed simply connected region, with the singularities of its analytic continuation. They establish that the errors taken in the Chebyshev sense on certain classes of rational functions whose members have $\nu$-poles in the complement of the region, define for $\nu=1,2, \ldots$ a sequence whose convergence rate determines a larger specified region of meromorphy along with the number of the (free) polar singularities of the analytic continuation therein. 
The present paper concerns extension of these results to axisymmetric potentials in $E^{3}$ as Fourier series of Zonal Harmonics on $E^{3}$. Specifically, using the Bergman [2] and Gilbert [6] Integral Operator Method an ASP whose initial domain of regularity is a closed sphere can be approximated in the Chebyshev sense over certain classes of Newtonian potentials each of whose members have $\nu$-singular circles in the complement of the sphere for $\nu=1,2, \cdots$. The convergence properties of the sequence of errors generated by the approximates determine the sphere and the number of (free) singular circles of the harmonic continuation in that sphere.

Function theoretic "transplants" of the classical theorems are thereby obtained. From these, information about the singularities of a solution to the axisymmetric LaPlace equation may then be obtained from information concerning the approximation of its boundary values. This is of interest in light of Gilbert's Method of Ascent [7] where solutions to more general classes of axisymmetric elliptic equations in $E^{3}$ are represented as the integral transforms of associated ASP with the property that the boundary values of the solution and associate are identical on certain spheres.

Moreover, by considering harmonic polynomials as certain Newtonian potentials whose principal parts have their singular circles located at infinity, these results complement necessary and sufficient conditions given by P. A. McCoy $[11,12]$ for a real GASP regular on a hypersphere and continuous on its closure to harmonically continue as an entire function GASP of specified (possibly infinite) order and (possibly zero) type. These were determined from properties of the sequence of errors in the Chebyshev approximation of the GASP on the closed hypersphere over classes of harmonic polynomials. That information was obtained by function theoretic methods from the classical theorem of S. N. Bernstein [3], including its generalizations by R. S. Varga [17] and A. R. Reddy [13, 14, 15] which deal with global existence and growth of real analytic functions via properties of local Chebyshev polynomial approximation. We now introduce the

2. Preliminary definitions and formulae. The instrument for this study is the invertible integral operator $W \equiv A_{1 / 2}$ [see 6] associating the unique function $f$,

$$
f(z)=\sum_{n=0}^{\infty} a_{n} z^{n}, \quad z=x+i y
$$

analytic in a neighborhood $\omega$ of the origin in the $C$-plane with the ASP $F$, 


$$
F(x, y)=\sum_{n=0}^{\infty} a_{n}\left(x^{2}+y^{2}\right)^{n / 2} P_{n}^{(0,0)}\left(x /\left(x^{2}+y^{2}\right)^{1 / 2}\right),
$$

$\left(P_{n}^{(0,0)}\right.$ are the Legendre polynomials [1]) regular in a neighborhood $\Omega$ of the origin in $E^{3}$ by

$$
F(x, y)=1 / 2 \pi i \int_{|\zeta|=1} f(\tau) \zeta^{-1} d \zeta
$$

with the vector $\tau$ defined as

$$
\tau=x+i y\left(\zeta+\zeta^{-1}\right) / 2 .
$$

The operator $W^{-1} \equiv A_{1 / 2}^{-1}$ [see 6], inverting $W$, associates with each ASP $F$ regular on $\Omega$ a unique $f$ analytic on $\omega$ by the transform

$$
f(z)=\int_{\xi=-1}^{\xi=+1} K\left(z r^{-1}, \xi\right) F\left(r \xi, r \sqrt{1-\xi^{2}}\right) d \xi
$$

where the principal branch of the kernel

$$
K(\eta, \xi)=\left(1-\eta^{2}\right) /\left(1-2 \xi \eta+\eta^{2}\right)^{3 / 2}
$$

is taken. The function elements $F$ and $f$ are defined on their first Riemann sheets over their respective domains of association. In other words, the global ASP $F$ and global associate $f$ are principal branches defined by harmonic, respectively analytic, continuation of the series in (1) and (2) from their initial domains of definition by the Envelope Method [6,7]. For the construction of the principal branches across surfaces of separation see [2, 6, p. 190ff.]. A fundamental global relation between the ASP $F$ and the associate $f$ was deduced by Gilbert [7, p. 18] who applied the Envelope Method to construct the singular sets of $W[f]$ and $W^{-1}[F]$ determining from their intersection that the necessary and sufficient condition for $F$ to be singular on the hypercircle $x+i y=\gamma$, is that the associate $f$ be singular at the point $z=\gamma$ or $z=\bar{\gamma}$.

The study of relations between these singularities and the approximation and harmonic continuation of ASP requires a potential theoretic analogy of rational functions $r_{n, \nu}$,

$$
r_{n, \nu}(z)=p_{n}(z) / q_{\nu}(z), \quad n, \nu=0,1,2, \cdots
$$

of type $(n, \nu)$. These are the ratio of relatively prime polynomials $p_{n}$ and $q_{\nu}$ of respective degrees $n$ and $\nu$ used by Saff and Walsh in their theory of rational approximation in the plane. This generalization employs *-quasimultiplication of ASP [see 6, p. 193ff.], a commutative, associative and distributive operation over addition, to define Newtonian potentials $R_{n, \nu}$ of type $(n, \nu)$ as 


$$
R_{n, \nu}=P_{n}^{*}\left(1 / Q_{\nu}\right)=W\left[r_{n, \nu}\right],
$$

$n, \nu=0,1,2, \cdots$ for the associate $r_{n, \nu}$. These include axisymmetric harmonic polynomials as Newtonian potentials $R_{n, 0}$ of type $(n, 0)$, $n=0,1,2, \cdots$. Moreover, a Newtonian potential of the type $(0,1)$ is the ASP generalization of a simple pole. The residue theorem shows that on the initial domain of definition,

$$
R_{0,1}(x, y, \gamma)=W\left[(z-\gamma)^{-1}\right]=\left[(x-\gamma)^{2}+y^{2}\right]^{-1 / 2}
$$

and that a $k$ th order pole corresponds to

$$
R_{0, k}(x, y, \gamma)=R_{0,1}(x, y, \gamma) * \ldots * R_{0,1}(x, y, \gamma)
$$

$k$-times, a potential

$$
R_{0, k}(x, y, \gamma)=1 /\left.(k-1) !(\partial / \partial \zeta)^{k-1} R_{0,1}(x, y, \zeta)\right|_{\zeta=r}
$$

with a singular circle of order $k$ at $x+i y=\gamma$. The form of the Newtonian potential of type $(n, \nu)$ is then

$$
R_{n, \nu}(x, y)=R_{n(\nu), 0}(x, y)+\sum_{j=0}^{\nu} b_{j} R_{0, m_{j}}\left(x, y, \gamma_{j}\right)
$$

for associates

$$
r_{n, \nu}(z)=p_{n(\nu)}(z)+\sum_{j=0}^{\nu} b_{j} /\left(z-\gamma_{j}\right)^{m_{j}}
$$

$m_{0}+m_{1}+\cdots+m_{\nu}=\nu$. On their domains of association, these formulae are interpreted as in earlier references. What is then meant by an ASP having a singular circle of order $\nu$ (counted with multiplicity) at $x+i y=\gamma_{j}$ is that the principal part of the Mittag-Leffler ASP expansion [see 6, p. 184],

$$
F(x, y)=\sum_{\nu=0}^{\infty} R_{n(\nu), \nu}(x, y)+E(x, y)
$$

$(E(x, y)$ is an entire function ASP) generated by the harmonic continuation process contains a term of the form (3) as in function theory. Such meromorphic ASP expansions are analogies of the Mittag-Leffler expansion

$$
f(z)=\sum_{\nu=0}^{\infty} r_{n(\nu), \nu}(z)+e(z)
$$

from the theory of analytic functions. We observe that the number of singular circles and the number of poles counted with multiplicity of the ASP and associate located within a specified distance of the origin again agree in view of the relation between expansions in (4) 
and (5). For representations of ASP with meromorphic associates, also see M. Marden [10].

To count the (free) singular circles of the harmonic continuation of ASP $F$ from its closed sphere of regularity $\Sigma_{\sigma}$ of radius $\sigma>0$ about the origin to a larger concentric sphere, requires a measure of the distances

$$
\left\|\mid F-R_{n, \nu}\right\|_{\sigma}=\sup \left\{\left|F(x, y)-R_{n, \nu}(x, y)\right|: x^{2}+y^{2}=\sigma^{2}\right\},
$$

$n, \nu=0,1,2, \cdots$ between $F$ and members $R_{n, \nu}$ of the sets $\mathscr{R}_{n, \nu}(\sigma)$ of Newtonian potentials of type $(n, \nu)$ regular in $\Sigma_{\sigma}$. The essential measures are drawn from the errors in the Chebyshev norm approximations,

$$
E_{n, \nu}(F)=\inf \left\{\left\||| F-R_{n, \nu}\right\|_{\sigma}: R_{n, \nu} \in \mathscr{R}_{n, \nu}(\sigma)\right\}
$$

$n, \nu=0,1,2, \cdots(\sigma$-fixed $)$ over the classes $\mathscr{R}_{n, \nu}(\sigma)$ which are analyzed by "transplanting" known error bounds found by Saff and Walsh for the associates. Namely, from their estimates of

$$
e_{n, \nu}(f)=\inf \left\{\left\|f-r_{n, \nu}\right\|_{\sigma}: r_{n, \nu} \in \mathfrak{r}_{n, \nu}(\sigma)\right\}
$$

and

$$
\left\|f-r_{n, \nu}\right\|_{\sigma}=\sup \left\{\left|f(z)-r_{n, \nu}(z)\right|:|z|=\sigma\right\}
$$

( $\sigma$-fixed) $n, \nu=0,1,2, \cdots$ where $\mathfrak{x}_{n, \nu}(\sigma)$ corresponds to " $W^{-1}\left[\mathscr{R}_{n, \nu}(\sigma)\right]$ ", designating all rational functions $r_{n, \nu}$ of type $(n, \nu)$ regular in the closed disk $D_{\sigma}$ of radius $\sigma$ about the origin. It is convenient to normalize the radius as $\sigma<1$, an assumption requiring no loss of generality because the LaPlace equation is invariant under homothetic transformations. Moreover, it is to be understood that the orders of the singular circles and poles of ASP and associate are counted with multiplicity. This brings us to the main objective which we treat in the next section.

3. Approximation and harmonic continuation. First, the degree of "rational" approximation by Newtonian potentials of an ASP over its sphere of regularity is related to the known number of singular circles which its harmonic continuation has within a larger concentric sphere of specified radius. This is principally a function theoretic transplant of J. L. Walsh's theorem [16, 19].

THEOREM 1. Let $F$ be an ASP regular on the sphere $\Sigma_{\sigma}$ whose harmonic continuation to the sphere $\Sigma_{\rho}(1 \leqq \rho \leqq \infty)$ has atmost $\nu$ singular circles. Then there is an approximating sequence of Newtonian potentials $R_{n, \nu}$ of respective types $(n, \nu)$ for which 


$$
\limsup _{n \rightarrow \infty}\left\|F-R_{n, \nu}\right\|_{\sigma}^{1 / n} \leqq \rho^{-1}
$$

Proof. Let ASP $F$, regular on the sphere $\Sigma_{\sigma}$, continue harmonically to the sphere $\Sigma_{\rho}$ with atmost $\nu$-singular circles. The associate $f$ is regular on the disk $D_{\sigma}$, a consequence of a theorem of Gilbert [7, p. 187] concerning the relationship between the singularities of the potential and the associate. Furthermore, the correspondence between the Mittag-Leffler expansions of meromorphic ASP and its meromorphic associate shows that the analytic continuation of $f$ to the disk $D_{\rho}$ has atmost $\nu$-poles. According to Walsh's result [16], there then exists an approximating sequence of rational functions $r_{n, \nu}$ of types $(n, \nu)$ with no poles in the disk $D_{o}$ for which

$$
\limsup _{n \rightarrow \infty}\left\|f-r_{n, \nu}\right\|_{\sigma}^{1 / n} \leqq \rho^{-1} \text {. }
$$

From the $W$-operator and the maximum principal

$$
F(x, y)-R_{n, \nu}(x, y)=W\left[f-r_{n, \nu}\right],
$$

$R_{n, \nu} \in \mathscr{R}_{n, \nu}(\sigma)$ one deduces the sequence of estimates

$$
\left|F(x, y)-R_{n, \nu}(x, y)\right| \leqq\left\|f-r_{n, \nu}\right\|_{\sigma}, \quad x^{2}+y^{2}<\sigma^{2}
$$

and

$$
\left\|F-R_{n, \nu}\right\|_{\sigma} \leqq\left\|f-r_{n, \nu}\right\|_{\sigma}, \quad n, \nu=0,1,2, \cdots
$$

so that we find

$$
\limsup _{n \rightarrow \infty}\left\|F-R_{n, \nu}\right\|_{\sigma}^{1 / n} \leqq \limsup _{n \rightarrow \infty}\left\|f-r_{n, \nu}\right\|_{\sigma}^{1 / n} .
$$

However, from the inverse operator, when

$$
f(z)-r_{n, \nu}(z)=W^{-1}\left[F-R_{n, \nu}\right]
$$

then

$$
\begin{gathered}
\left|f(z)-r_{n, \nu}(z)\right| \leqq \mu(\sigma)|| F-R_{n, \nu} \|_{\sigma^{\prime}}|z|<\sigma \\
\mu(\sigma)=\sup \left\{\int_{-1}^{+1}|K(z, \xi)| d \xi:|z|<\sigma\right\}
\end{gathered}
$$

where $\mu(\sigma) \leqq 1$ for $\sigma<1$. This leads to the appraisals

$$
\left\|f-r_{n, \nu}\right\|_{o}^{1 / n} \leqq[\mu(\sigma)]^{1 / n}\left\|F-R_{n, \nu}\right\|_{o}^{1 / n}
$$

and

$$
\limsup _{n \rightarrow \infty}\left\|f-r_{n, \nu}\right\|_{\sigma}^{1 / n} \leqq \limsup _{n \rightarrow \infty}\left\|F-R_{n, \nu}\right\|_{\sigma}^{1 / n} .
$$

Combination of the inequalities (11) and (14) completes the proof of 
the Walsh type approximation theorem for ASP.

Our next consideration is the converse of Theorem 1, namely the function theoretic "transplant" of the theorem of Saff [16]. It is the characterization of the singularities of the harmonic continuation found in Theorem 2.

THeOREM 2. Let the ASP $F$ be regular on the sphere $\Sigma_{\sigma}$. If an approximating sequence of Newtonian potentials $R_{n, \nu}$ of respective types $(n, \nu)$ exists on the sphere $\Sigma_{o}$ for which

$$
\limsup _{n \rightarrow \infty}\left\|F-R_{n, \nu}\right\|_{\sigma}^{1 / n} \leqq \rho^{-1}(<1),
$$

then $F$ can be harmonically continued to the sphere $\Sigma_{\rho}$ with atmost ע-singular circles.

Proof. Let the ASP $F$ be regular on the sphere $\Sigma_{\sigma}$ and be approximated by a sequence of Newtonian potentials $R_{n, \nu}$ of types $(n, \nu)$ on the sphere $\Sigma_{\sigma}$ satisfying the required bound. The associates of $F$ and of the members $R_{n, \nu}$ of the sequence, $f=W^{-1}[F]$ and $r_{n, \nu}=W^{-1}\left[R_{n, \nu}\right]$, are regular functions on the disk $D_{\sigma}$ by [16]. Reasoning as in (12-14) then gives the bound on the associate,

$$
\limsup _{n \rightarrow \infty}\left\|f-r_{n, \nu}\right\|_{o}^{1 / n} \leqq \rho^{-1}
$$

which is found in Saff's Theorem 2. Then $f$ may be analytically continued from the disk $D_{\sigma}$ to the disk $D_{\rho}$ as an analytic function with atmost $\nu$-singularities. Properties of the $W$-transform considered earlier show that the ASP $F$ has a harmonic continuation to the sphere $\Sigma_{\rho}$ with atmost $\nu$-singular circles.

The main theorem we now come to characterizes the singular circles of the harmonic continuation of an ASP within a given distance of its initial sphere of regularity. This is a transformation of Saff's theorem on continuation properties of analytic functions.

Theorem 3. Let the ASP $F$ be regular on the sphere $\Sigma_{\sigma}$ and for each $\nu$ let the measure of the error in the Chebyshev approximation of $F$ over the sets of Newtonian potentials of types $(n, \nu)$ be given by

$$
\rho_{\nu}^{-1} \equiv \limsup _{n \rightarrow \infty}\left[E_{n, \nu}(F)\right]^{1 / n}, \quad \nu=0,1,2, \cdots .
$$

Then the sequence $\left\{\rho_{\nu}\right\}_{\nu=0}^{\infty}$ is nondecreasing and

(i) the sphere of largest radius in which $F$ harmonically continuous as an ASP with $\nu$-singular circles is $\Sigma_{\rho_{\nu}}$. If $\rho_{\nu}>\rho_{\nu-1}$, the harmonic continuation of $F$ has precisely $\nu$-singular circles in $\Sigma_{\rho_{\nu}}$. 
(ii) the largest sphere $\Sigma_{\rho}$ in whose interior $F$ may be harmonically continued as an (meromorphic) ASP has radius $\rho=\lim _{\nu \rightarrow \infty} \rho_{\nu}$.

(iii) the harmonic continuation of the ASP $F$ has an essential singularity on the boundary of the sphere $\Sigma_{\rho}$, if and only if, $\rho_{\nu}<\rho$ for $\nu=0,1,2, \cdots$.

Proof. Let the ASP $F$ be regular on the sphere $\Sigma_{\sigma}$ and let $\left\{R_{n, 2}\right\}_{n, \nu=0}^{\infty}$ be an approximating sequence of Newtonian potentials on $\Sigma_{\sigma}$. Then from the $W$-transform

$$
\left\|F-R_{n, \nu}\right\|_{\sigma} \leqq\left\|f-r_{n, \nu}\right\|_{\sigma}
$$

and

$$
\limsup _{n \rightarrow \infty}\left[E_{n, \nu}(F)\right]^{1 / n} \leqq \limsup _{n \rightarrow \infty}\left[e_{n, \nu}(f)\right]^{1 / n}
$$

for $\nu=0,1,2, \cdots$. However, in (13) the inverse operator gave

$$
\left\|f-r_{n, \nu}\right\|_{\sigma}^{1 / n} \leqq[\mu(\sigma)]^{1 / n}\left\|F-R_{n, \nu}\right\|_{\sigma}^{1 / n}
$$

Then the estimate

$$
\left[e_{n, \nu}(f)\right]^{1 / n} \leqq[\mu(\sigma)]^{1 / n}\left\|F-R_{n, \nu}\right\|_{\sigma}^{1 / n}
$$

leads to

$$
\limsup _{n \rightarrow \infty}\left[e_{n, \nu}(f)\right]^{1 / n} \leqq \limsup _{n \rightarrow \infty}\left[E_{n, \nu}(F)\right]^{1 / n}
$$

so that

$$
\limsup _{n \rightarrow \infty}\left[e_{n, \nu}(f)\right]^{1 / n}=\limsup _{n \rightarrow \infty}\left[E_{n, \nu}\left(F^{\prime}\right)\right]^{1 / n}
$$

for $\nu=0,1,2, \cdots$. The Saff theorem shows that if

$$
\rho_{\nu}^{-1}=\limsup _{n \rightarrow \infty}\left[e_{n, \nu}(f)\right]^{1 / n}, \quad \nu=0,1,2, \cdots
$$

then the sequence $\left\{\rho_{\nu}\right\}_{\nu=0}^{\infty}$ is nondecreasing. We now proceed with this sequence to complete the characterizations of the harmonic continuation.

(i) Let the sphere of largest radius in which the harmonic continuation of $F$ has atmost $v$-singular circles be $\Sigma_{\eta}$. To determine its radius, observe that by the correspondence between the singularities and poles of the Mittag-Leffler expansions of ASP and associate, the associate has atmost $\nu$-poles in the disk $D_{\eta}$. By Saff's result, $\eta=\rho_{\nu}$ which completes the first part. Moreover, if $\rho_{\nu-1}<\rho_{\nu}$ the associate has precisely $\nu$-poles in the disk $D_{\rho_{\nu}}$ which correspond to $\nu$-singular circles in the sphere and conversely.

(ii) Let the sphere of largest radius in which $F$ harmonically 
continues as a meromorphic ASP by the Envelope Method be $\Sigma_{\rho}$. The maximal disk of meromorphy for the associate is accordingly $D_{\rho}$ since analytic continuation of $f$ to a larger disk would allow harmonic continuation of $F$ beyond $\Sigma_{\rho}$ by the Envelope Method. It is a consequence of [16] that the radius of $D_{\rho}$ is $\rho=\lim _{\nu \rightarrow \infty} \rho_{\nu}$.

(iii) The fact that the associate has infinitely many poles on the boundary of the disk $D_{\rho}$, if and only if, the ASP has infinitely singular circles on the boundary of the sphere $\Sigma_{\rho}$ follows as an immediate consequence of the theorem of Gilbert. Saff's theorem shows that the associate has infinitely many singularities on $\partial D_{\rho}$, if and only if, $\rho_{\nu}<\rho$ for $\nu=0,1, \cdots$.

We now consider

4. Generalizations. For the integers $2 \alpha=k-3, k=3,4, \cdots$ the regular Fourier series expansion

$$
F^{\alpha}(x, y)=\sum_{n=1}^{\infty} a_{n}\left(x^{2}+y^{2}\right)^{n / 2} P_{n}^{(\alpha, \alpha)}\left(x /\left(x^{2}+y^{2}\right)^{1 / 2}\right) / P_{n}^{(\alpha, \alpha)}(1),
$$

(the $P_{n}^{(\alpha, \alpha)}$ are the ultraspherical polynomials [1]) in a neighborhood of the origin is the unique representation of a GASP in $E^{2 \alpha}$. Using an alternate form of the $A_{\mu}$ operator [6, p. 168], $W_{\alpha}[11]$, analytic associates (1) are mapped onto (18) by the operator

$$
\begin{aligned}
& F^{\alpha}(x, y)=W_{\alpha}[f]=\int_{|\zeta|=1} f(\tau) d \lambda_{\alpha}(\zeta) \\
& d \lambda_{\alpha}(\zeta)=c_{\alpha}\left(\zeta-\zeta^{-1}\right)^{2 \alpha} d \zeta / \zeta \\
& c_{\alpha}^{-1}=\int_{|\zeta|=1} d \lambda_{\alpha}(\zeta) .
\end{aligned}
$$

An application of the residue theorem shows the GASP generalization of a simple pole (on its initial domain of definition) is given by

$$
\begin{aligned}
& R_{0,1}^{\alpha}(x, y, \gamma)=W_{\alpha}\left[(z-\gamma)^{-1}\right] \\
& \quad=\left[(x-\gamma)^{2}+y^{2}\right]^{(2 \alpha-1) / 2} /(i y)^{2 \alpha}+\operatorname{sgn}(2 \alpha) S_{\alpha}(x, y, \gamma), \\
& S_{\alpha}(x, y, \gamma)=\sum \frac{\Gamma(2 \alpha) \Gamma(2 \alpha)}{\left(n_{1} !\right)^{2}\left(n_{2} !\right)^{2}}\left[\frac{(x-\gamma)+\left((x-\gamma)^{2}+y^{2}\right)^{1 / 2}}{i y}\right]^{n_{3}-n_{4}}
\end{aligned}
$$

where the sum is taken on nonnegative integers for which $n_{1}+n_{2}+n_{3}+n_{4}=2 \alpha-1$. The GASP generalization of a pole of order $k$ is then defined by *quasimultiplication

$$
F_{1}^{\alpha} * F_{2}^{\alpha}=W_{\alpha}\left[f_{1} f_{2}\right]
$$

as

$$
R_{0, k}^{\alpha}(x, y, \gamma)=R_{0,1}^{\alpha}(x, y, \gamma) * \cdots * R_{0,1}^{\alpha}(x, y, \gamma)
$$


$k$-times so that

$$
R_{0, k}^{\alpha}(x, y, \gamma)=\left.(\partial / \partial \zeta)^{k-1} R_{0,1}^{\alpha}(x, y, \zeta)\right|_{\zeta=r} / \Gamma(k) .
$$

These functions form the basis for the principal part of the MittagLeffler GASP expansion. The difficulty, however, in applying these expansions to extend the classical theorems to GASP is in the complexity with which the principal branch of the harmonic continuation must be defined across surfaces of separation. It should be further noted that for nonintegers $2 \alpha>0, W_{\alpha}\left[(z-\gamma)^{-1}\right]$ may be evaluated by Legendre functions as in Gilbert [6] or by the contour integral methods in [9]. Finally the case that $2 \alpha<0$ need not be considered, as D. Colton [4] has shown that the Dirichlet problem need not be unique in this range of $\alpha$ due to nontrivial representations of zero.

Approximation and harmonic continuation of ASP from initial domains which are not spherical leads us to consider axisymmetric sets $\Omega \subset E^{3}$ which are generated by rotating axiconvex sets $\omega \subset C$ into $E^{3}$ about the axis of symmetry. An axiconvex set $\omega$ is a simply connected set with the property that $\zeta \in \omega$, if and only if, $\operatorname{seg}\{\zeta, \bar{\zeta}\} \in \omega$. If the locus $\Gamma_{\sigma}(\sigma>1)$ taken relative to $\omega$ as in the Saff theorem is an axiconvex set, the norms $(6,7)$ and $(8,9)$ previously taken (after a homothetic transformation) on spheres and circles may be redefined on $\Omega$ and $\omega$ to include harmonic/analytic continuation from these initial domains to axisymmetric or axiconvex domains of association. However, we know of no sufficient condition on the maps $\phi$ as required in Saff's paper for the set $\Gamma_{\sigma}=\partial \omega$ to be axiconvex as required for such an extension.

ACKNOWLEDGMENT. This research was partially supported by a grant from the U. S. Naval Academy Research Council.

\section{REFERENCES}

1. R. Askey, Orthogonal polynomials and special functions, Regional Conference Series in Applied Math., SIAM, Philadelphia, 1975.

2. S. Bergman, Integral operators in the theory of linear partial differential equations, Ergebnisse der Mathematik und ihrer Grenzebiete, Heft 23, Springer-Verlag, New York, 1961.

3. S. N. Bernstein, Lecon sur les propriétés extrémales et la meillure approximation des functions analytiques d'une variable ree'lle, Gauthier-Villars, Paris, 1926.

4. D. Colton, Applications of a class of singular partial equations to Gegenbauer series which converge to zero, SIAM J. Math. Anal., 1, no. 1, (1970), 90-95.

5. P. Dienes, The Taylor Series, Dover Publications, New York, 1957.

6. R. P. Gilbert, Function theoretic methods in partial differential equations, Math. in Science and Engineering, vol. 54, Academic Press, New York, 1969.

7. Constructive methods for elliptic equations, Lecture Notes in Mathematics, vol. 365, Springer-Verlag, New York, 1974. 
8. J. Hadamard, Essai sur l'etude des fonctions données par leur developpement de Taylor, in Oeuvres de Jacques Hadamard. vol. 1, Centre National de la Recherche Scientifique, Paris, 1968.

9. J. L. Lavoie, T. J. Osler and R. Tremblay, Fractional derivatives and special functions, SIAM Review, 18, no. 2, (1976), 240-268.

10. M. Marden, Axisymmetric vectors, Amer. J. Math., 67, (1945), 109-122.

11. P. A. McCoy, Polynomial approximation and growth of generalized axisymmetric potentials, Canad. J. Math., 31 (1979), 49-59.

12. — Polynomial approximation of generalized biaxisymmetric potentials, J. Approx. Theory, in press.

13. A. R. Reddy, Approximation of an entire function, J. Approx. Theory, no. 3, (1970), 128-137.

14. Best polynomial approximation of certain entire functions, J. Approx. Theory, no. 5, (1972), 97-112.

15. - Addendum to "Best polynomial approximation of certain entire functions," J. Approx. Theory, no. 12, (1974), 199-200.

16. E. B. Saff, Regions of meromorphy determined by the degree of best rational approximation, Proc. Amer. Math. Soc., 29, no. 1, (1971), 30-38.

17. R. S. Varga, On an extension of a result of $S$. N. Bernstein, J. Approx. Theory, (1968), 176-179.

18. J. L. Walsh, Interpolation and approximation by rational functions in the complex domain, 3rd ed., Amer. Math. Soc. Colloq. Publ. vol. 20, Providence, R. I., 1960.

19. - The convergence of sequences of rational functions of best approximation with some free poles, Proc. Sympos. Approximation of Functions (General Motors Res. Lab., 1964), Elsevier, Amsterdam, (1965), 1-16.

Received May 31, 1978. This research was partially supported by Department of Mathematics.

U. S. Naval ACADEMY

ANNAPOLIS, MD 21402 



\title{
PACIFIC JOURNAL OF MATHEMATICS
}

\section{EDITORS}

\author{
DONALD BABBITT (Managing Editor) \\ University of California \\ Los Angeles, CA 90024 \\ HUGo ROSSI \\ University of Utah \\ Salt Lake City, UT 84112 \\ C. C. MOORE \\ University of California \\ Berkeley, CA 94720
}

J. DugundJI

Department of Mathematics

University of Southern California

Los Angeles, CA 90007

R. FinN and J. Milgram

Stanford University

Stanford, CA 94305

\section{ASSOCIATE EDITORS}
E. F. BECKENBACH
B. H. Neumann
F. WolF
K. YOSHIDA

\section{SUPPORTING INSTITUTIONS}

\author{
UNIVERSITY OF BRITISH COLUMBIA \\ CALIFORNIA INSTITUTE OF TECHNOLOGY \\ UNIVERSITY OF CALIFORNIA \\ MONTANA STATE UNIVERSITY \\ UNIVERSITY OF NEVADA, RENO \\ NEW MEXICO STATE UNIVERSITY \\ OREGON STATE UNIVERSITY \\ UNIVERSITY OF OREGON
}

UNIVERSITY OF SOUTHERN CALIFORNIA

STANFORD UNIVERSITY

UNIVERSITY OF HAWAII

UNIVERSITY OF TOKYO

UNIVERSITY OF UTAH

WASHINGTON STATE UNIVERSITY

UNIVERSITY OF WASHINGTON

The Supporting Institutions listed above contribute to the cost of publication of this Journal, but they are not owners or publishers and have no responsibility for its content or policies.

Mathematical papers intended for publication in the Pacific Journal of Mathematics should be in typed form or offset-reproduced, (not dittoed), double spaced with large margins. Please do not use built up fractions in the text of the manuscript. However, you may use them in the displayed equations. Underline Greek letters in red, German in green, and script in blue. The first paragraph or two must be capable of being used separately as a synopsis of the entire paper. Please propose a heading for the odd numbered pages of less than 35 characters. Manuscripts, in triplicate, may be sent to any one of the editors. Please classify according to the scheme of Math. Reviews, Index to Vol. 39. Supply name and address of author to whom proofs should be sent. All other communications should be addressed to the managing editor, or Elaine Barth, University of California, Los Angeles, California, 90024.

50 reprints to each author are provided free for each article, only if page charges have been substantially paid. Additional copies may be obtained at cost in multiples of 50 .

The Pacific Journal of Mathematics is issued monthly as of January 1966. Regular subscription rate: $\$ 72.00$ a year (6 Vols., 12 issues). Special rate: $\$ 36.00$ a year to individual members of supporting institutions.

Subscriptions, orders for numbers issued in the last three calendar years, and changes of address should be sent to Pacific Journal of Mathematics, P.O. Box 969, Carmel Valley, CA 93924, U.S.A. Older back numbers obtainable from Kraus Periodicals Co., Route 100, Millwood, NY 10546.

PUBLISHED BY PACIFIC JOURNAL OF MATHEMATICS, A NON-PROFIT CORPORATION

Printed at Kokusai Bunken Insatsusha (International Academic Printing Co., Ltd.). 8-8, 3-chome, Takadanobaba, Shinjuku-ku, Tokyo 160, Japan.

Copyright (C) 1979 by Pacific Journal of Mathematics Manufactured and first issued in Japan 


\section{Pacific Journal of Mathematics}

\section{Vol. 81, No. $2 \quad$ December, 1979}

Ersan Akyildiz, Vector fields and equivariant bundles ............... 283

Ehrhard Behrends, The centralizer of tensor products of Banach spaces ( $a$ function space representation) ......................... 291

Geoffrey R. Burton, Congruent sections of a convex body ............. 303

John Warnock Carlson, $H$-closed and countably compact extensions ...... 317

Robert Charles Carlson, Eigenfunction expansions for selfadjoint

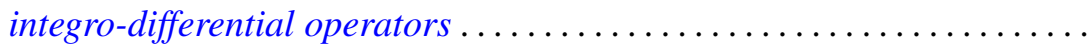

Robert Damiano, Coflat rings and modules.

Eric Karel van Douwen and Washek (Vaclav) Frantisek Pfeffer, Some properties of the Sorgenfrey line and related spaces ................

Uri Elias, Necessary conditions and sufficient conditions for disfocality and

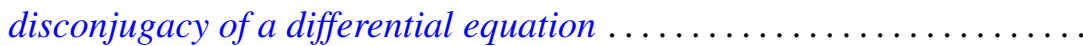

V. L. (Vagn Lundsgaard) Hansen, Polynomial covering spaces and homomorphisms into the braid groups .......................

Paul Hess, Dedekind's problem: monotone Boolean functions on the lattice

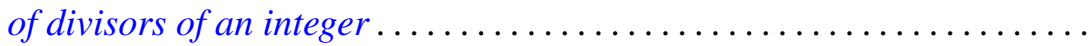

Alan Hopenwasser and David Royal Larson, The carrier space of a reflexive

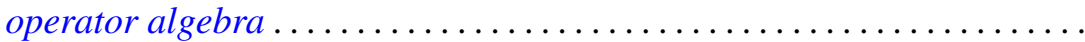

Kyung Bai Lee, Spaces in which compacta are uniformly regular $G_{\delta} \ldots \ldots$.

Claude Levesque, A class of fundamental units and some classes of

Jacobi-Perron algorithms in pure cubic fields...

Teck Cheong Lim, A constructive proof of the infinite version of the

Belluce-Kirk theorem ..........................

Dorothy Maharam and A. H. Stone, Borel boxes

Roger McCann, Asymptotically stable dynamical systems are linear

Peter A. McCoy, Approximation and harmonic continuation of axially symmetric potentials in $E^{3} \ldots \ldots \ldots \ldots \ldots \ldots \ldots$

Takahiko Nakazi, Extended weak-* Dirichlet algebras ....

Carl L. Prather, On the zeros of derivatives of balanced trigonometric polynomials ................................

Iain Raeburn, An implicit function theorem in Banach spaces...

Louis Jackson Ratliff, Jr., Two theorems on the prime divisors of zeros in completions of local domains...

Gloria Jean Tashjian, Cartesian-closed coreflective subcategories of

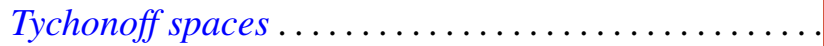

Stephen Edwin Wilson, Operators over regular maps.... . . 